\title{
Patrones funcionales del vocativo en la producción narrativa de Benito Pérez Galdós*
}

\author{
Functional patterns of the vocative in the narrative production \\ of Benito Pérez Galdós
}

Guadalupe Nieto Caballero**

\begin{abstract}
RESUMEN
En este artículo se presenta el uso que hace Benito Pérez Galdós de los vocativos como eje de un patrón estructural con el que el escritor consigue crear diversos efectos literarios significativos. Nos hemos centrado en aquellos casos en los que una proposición proyectora va seguida de un vocativo (pronunciado por un personaje). Para ello hemos recurrido a un corpus formado por las novelas de Galdós, que hemos comparado con otro de referencia constituido por algunas novelas decimonónicas españolas. Como se verá, el patrón no solo se usa con más frecuencia en las novelas de Galdós, sino que el escritor lo usa con unos fines estilísticos concretos como crear una impresión de sincronía entre el habla de los personajes y su lenguaje corporal o proporcionar información acerca de los personajes. El análisis pretende ser una contribución al ámbito aún emergente de la estilística de corpus en español, ilustrando cómo el análisis de obras literarias puede beneficiarse ampliamente del manejo de herramientas innovadoras de corpus.
\end{abstract}

\section{SUMMARY}

This article presents Benito Pérez Galdós' use of vocatives as the axis of a structural pattern with which the writer manages to create various significant literary effects. We have focused on those cases in which a projecting proposition is followed by a vocative (pronounced by a character). For this we have re-
Palabras clave: estilística de corpus, Galdós, literatura española, vocativo.
Keywords: corpus stylistics, Galdós, Spanish literature, vocative.

\footnotetext{
* El artículo se enmarca en una amplia línea de investigación desarrollada por la autora.

** Española. Doctora en Lenguas y culturas por la Universidad de Extremadura. Académica Universidad de Extremadura. Cáceres, España. gnieto@unex.es
} 
sorted to a corpus made up of Galdós's novels, which we have compared with another corpus as reference made up of several nineteenth-century Spanish novels. As will be seen, the pattern is not only used more frequently in Galdós' novels, but the writer uses it for specific stylistic purposes such as creating an impression of synchronicity between the characters' speech and their body language or providing information about the characters. The analysis is intended to be a contribution to the still emerging field of corpus stylistics in Spanish, illustrating how the analysis of literary works can greatly benefit from the use of innovative corpus tools. 


\section{Introducción}

En el género narrativo, el vocativo suele ser parte del discurso de los personajes y la obra de Benito Pérez Galdós no es una excepción a esta tendencia. Sin embargo, en el uso que hace de este elemento el autor español se advierte, además, una clara relación entre el vocativo identificado en la intervención del personaje y el narrador que introduce este discurso. En concreto, el vocativo suele situarse como parte del parlamento del personaje precediendo a la proposición proyectora ${ }^{1}$ que sirve para introducir este discurso, como se muestra en el ejemplo (1), donde como vemos, el doble uso del vocativo "Don Ramón" se antepone a la proposición proyectora que contiene una referencia al sorbo de café que el personaje da antes de pronunciar su intervención discursiva:

(1) -Don Ramón, D. Ramón - dijo el elegante, que acababa de paladear su café-. ¿No sabe? A Cañizares, ¿ise acuerda usted?, el que estaba en Propiedades, aquel a quien llamábamos don Simplicio, le han dado los doce mil. ¿Ha visto usted polacada mayor? [Miau, capítulo 21 $]^{2}$

En Galdós, como se verá en este artículo, esta relación entre proposiciones proyectoras con referencias al lenguaje gestual de los personajes y sus vocativos introductores no solo es estadísticamente más frecuente que en la novela decimonónica española en general, sino que igualmente su uso es estilísticamente significativo y sirve para crear distintos efectos, como la simultaneidad entre las palabras del personaje y su lenguaje corporal, la caracterización e incluso la construcción del universo ficticio que nos plantea el autor.

Para llevar a cabo el estudio hemos utilizado un enfoque de estilística de corpus ${ }^{3}$, caracterizado por emplear metodologías propias de los estudios de lingüística de corpus en el análisis de textos literarios,

1 Siguiendo la nomenclatura propia de la gramática sistémica funcional, en este trabajo se utilizan los conceptos de proposición proyectora y proposición proyectada (Halliday, 2004) para referirse a la voz del narrador y a las palabras de los personajes respectivamente.

2 Todos los ejemplos citados en el artículo han sido extraídos de una versión electrónica de los textos (véase apartado 2). Por lo tanto, en lugar de la paginación, únicamente se ofrece como referencia el capítulo en el que aparecen.

3 Mahlberg $(2014,2016)$ y McIntyre y Walker (2019). 
con el fin de identificar "patrones de los que nosotros, como lectores, no somos conscientes, aunque estos pueden contribuir a los efectos que percibimos" $^{\prime 4}$ (Mahlberg, 2013, p. 27). En nuestro caso, como ya indicamos más arriba, hemos identificado el antedicho patrón en un corpus compuesto por las novelas de Galdós, constituido por un total de 6,3 millones de palabras, mientras en nuestro corpus de referencia de novela española decimonónica hemos obtenido un recuento de 6,1 millones de palabras. Esta comparación nos ha servido en un primer momento para evaluar el empleo que hace Galdós de este patrón, proceso en el cual hemos afrontado una visión analítica.

Antes de cerrar estas líneas de introducción, conviene incidir en que el vocativo es un elemento lingüístico ampliamente analizado en términos generales (Brioso Sánchez, 1971; Bañón Hernández, 1993; Alonso-Cortés, 1999; Edeso Natalías, 2005; entre otros). Tradicionalmente, su análisis ha girado en torno a cuestiones de tipo pragmático (Leech, 1983) y más concretamente de cortesía (Bravo y Briz, 2004). Y sobre estos aspectos se han llevado a cabo análisis de aplicación práctica, como en el discurso político (Cuenca, 2013). En el caso de la literatura también existen análisis variados, tanto en español - respecto de la figura de Bécquer (Balbín, 1968) — como en el ámbito internacional -Shakespeare (Busse, 2006) o las comedias de Plauto en el ámbito del mundo clásico (Monserrat Roig, 2009)—. Nuestro artículo, sin embargo, se aleja de este tipo de análisis, pues no nos ocupamos del valor discursivo del vocativo, sino de su función como elemento estructural en el marco del estilo de Galdós (Pattison, 1954), que nos ayudará a comprender mejor el proceso creativo del autor canario.

\section{Corpus de estudio y corpus de referencia}

Tanto los textos del corpus de estudio novelístico galdosiano (en adelante, CorBPG) como el de referencia novelístico decimonónico español (CorXIX) han sido descargados del repositorio digital de la Biblioteca Virtual Miguel de Cervantes (Universidad de Alicante, 2019). En el caso de CorBPG, hemos seleccionado todas sus novelas, con una ex-

4 La cita original es como sigue: "patterns that we as readers may not be aware of, although such patterns might still contribute to the effects we perceive". La traducción es nuestra. 
tensión que sobrepasa los 6,3 millones de palabras. Naturalmente, su producción teatral no ha sido incluida en el corpus por no pertenecer al género narrativo. Por una cuestión de consistencia metodológica, tampoco hemos tenido en cuenta su narrativa breve, por no tratarse de novelas propiamente dichas.

El CorXIX, en tanto, está formado por ochenta novelas de ocho autores diferentes: Pedro Antonio de Alarcón, Vicente Blasco Ibáñez, Leopoldo Alas Clarín, Luis Coloma, Armando Palacio Valdés, Emilia Pardo Bazán, José María de Pereda y Juan Valera, cuya extensión es similar a la que encontramos en CorBPG, con 6.188.478 palabras ${ }^{5}$. Para construir nuestro corpus de referencia, hemos seguido las nociones de representatividad y pertinencia de los textos propias de los estudios de lingüística de corpus en general, de tal suerte que constituya una muestra de la literatura realista española lo suficientemente sólida como para poder realizar una comparación cabal de los resultados obtenidos en el corpus galdosiano y establecer su representatividad antes de analizarlos ${ }^{6}$.

\section{Metodología y resultados}

Para realizar el análisis, hemos empleado el software de concordancias WordSmith Tools (Scott, 2013). En primer lugar, hemos localizado ejemplos de vocativos por separado en CorBPG y en CorXIX con el fin de realizar una comparación cabal y calibrar el uso que Galdós hace de este elemento en relación con la pléyade de autores con los que compartió plana. Para localizar los ejemplos en ambos corpus, hemos realizado la concordancia - dijo (guion + pretérito perfecto simple del verbo decir) en cada uno de ellos ${ }^{7}$. Esto es así porque tanto esta forma verbal como este tiempo son los más empleados para proyectar discurso (Nieto Caballero, 2018) y ese es el patrón estructural en el que aparecen los vocativos con frecuencia, donde el guion marca la barrera entre el estilo directo del personaje (en donde se encuentra el vocativo)

5 Pueden consultarse ambos corpus, con fechas de publicación y número de palabras totales, en Nieto Caballero (2019).

6 Para una información más detenida de las nociones de representatividad y pertinencia de CorXIX, véase Nieto Caballero (2018), en donde se explican los pormenores teóricos que guían la compilación de este corpus de referencia.

$7 \quad$ Por cuestiones de espacio, nos centramos únicamente en el verbo decir, que es el verbo de habla por excelencia, y no en otros verbos de comunicación. 
y el discurso del narrador que lo introduce, como se puede observar en (2). En la Figura 1 se muestra una captura de pantalla de esta concordancia en CorBPG.

(2) - Soy yo, señora - dijo Pepet en voz baja - soy yo, que aun aquí, donde está la monja más segura, vengo sin temor a nada, ni a la misma muerte. [Un voluntario realista, capítulo 29]

Figura 1. Captura de pantalla de WordSmith Tools de la concordancia-dijo

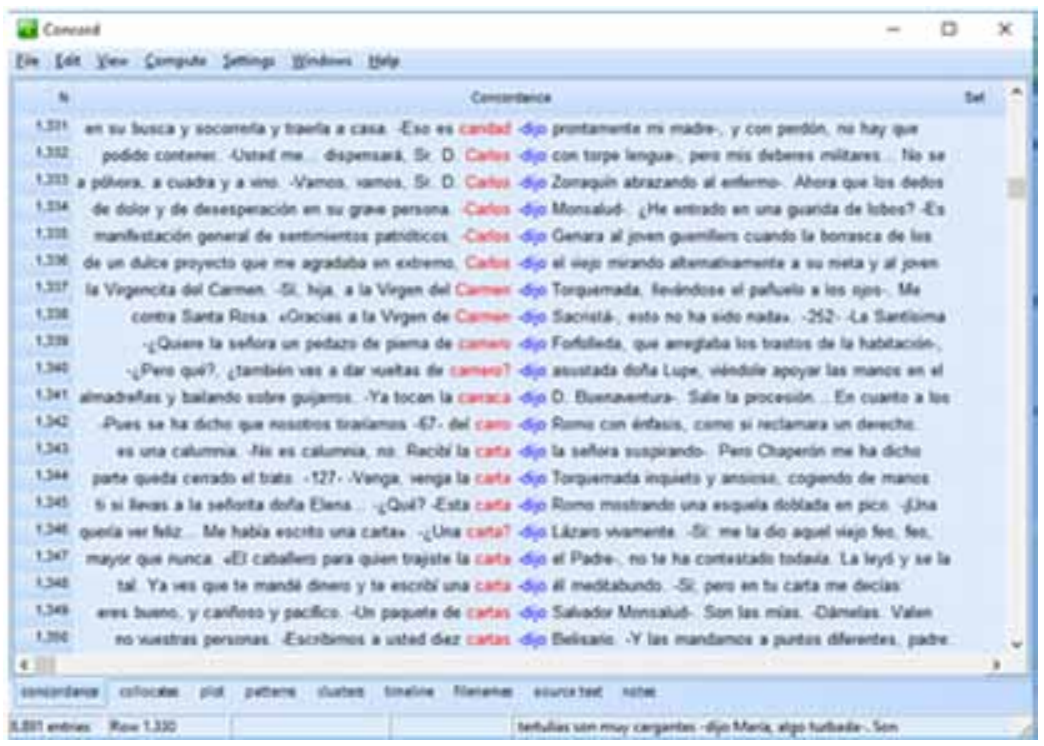

Esta concordancia rescata un total de 8.891 ejemplos en CorBPG (véase esquina inferior izquierda de la imagen) y 2.176 en CorXIX. Naturalmente, no todos los ejemplos de -dijo localizados tienen por qué estar precedidos de discurso en los que se emplee un vocativo. En la Figura 1, por ejemplo, la concordancia 1.331 no tiene vocativo, mientras que las 1.332, 1.333, 1.334, 1.335 y 1.336 sí. Para localizar los vocativos en ambas concordancias hemos hecho una búsqueda manual. Los resultados obtenidos aparecen recogidos en la Tabla 1. En términos absolutos, como se puede observar, existe un mayor número de vocativos en CorBPG (1.567) que en CorXIX (439), que no es sino el resultado del mayor número de ejemplos localizados en primera instancia. En otras palabras, si normalizamos estas frecuencias por cada cien ejemplos, observamos que en realidad el uso es similar. De hecho, en CorXIX comprobamos una frecuencia ligeramente mayor $(20,2 \%$ de los ejem- 
plos) que en CorBPG (17,6\%). Esto es, si bien Galdós proyecta mucho más el discurso de sus personajes utilizando estilo directo, el uso de vocativos como tal no es en realidad una estrategia más extendida en sus novelas.

Tabla 1

Proposiciones proyectoras localizadas con la concordancia -dijo

\begin{tabular}{lll}
\hline & CorBPG & CorXIX \\
\hline Proposiciones proyectoras (total) & 8.903 & 2.176 \\
\hline Proposiciones proyectoras que contienen vocativo & 1.567 & 439 \\
\hline \% proposiciones proyectoras que contienen vocativo & 17,6 & 20,2 \\
\hline
\end{tabular}

Fuente: Elaboración propia.

Sin embargo, en los ejemplos localizados se advierte un uso particular de estos vocativos. Formalmente, la proposición proyectora aparece con frecuencia interrumpiendo la proposición proyectada, partiendo esta en dos, como se puede observar en el ejemplo (3). Este tipo de proposición que interrumpe el parlamento del personaje en la estrategia de estilo directo se denomina suspensión (del inglés suspension), y puede definirse como "una interrupción prolongada del discurso del personaje por parte del narrador" (Lambert, 1981, p. 6) . $^{8}$ De las 1.567 proposiciones que contienen vocativo en CorBPG, se han localizado 1.341 que siguen esta pauta (un $85 \%$ ). En CorXIX, la cifra, aunque igualmente alta, desciende sensiblemente hasta un $64 \%$. La comparativa puede observarse en la Tabla 2 . El uso de suspensiones puede ser el resultado de la influencia que Dickens ejerció en Galdós, pues se trata de un elemento característico del estilo del novelista victoriano (Newsom, 2000). Su influencia en el autor español es ampliamente conocida por la crítica en general (Levy, 1937; McGovern, 2000; Tambling, 2013) y ha sido analizada de forma más concreta a través de este elemento recientemente (Nieto Caballero, 2019a).

(3) -Vamos, señor don Elías- dijo este descontento-. ¿Qué hago yo con cinco onzas? [La Fontana de Oro, capítulo 2]

8 La cita original es la siguiente: "a protracted interruption by the narrator of a character's speech". La traducción es nuestra. 
Tabla 2

Distribución de proposiciones proyectoras que contienen vocativos

\begin{tabular}{lllll}
\hline & CorBPG & $\%$ & CorXIX & $\%$ \\
\hline Suspensiones & 1.341 & 85,6 & 284 & 64,7 \\
\hline Patrón habitual & 226 & 14,4 & 155 & 35,3 \\
\hline Total & 1.567 & 100 & 439 & 100 \\
\hline
\end{tabular}

Fuente: Elaboración propia.

Como sostiene Lambert (1981), precisamente en su análisis acerca del uso de suspensiones en las novelas de Charles Dickens, estas son un lugar habitual para que el narrador, al proyectar el discurso de sus personajes, ofrezca al lector información respecto del modo de articulación de las palabras, su actitud e incluso el lenguaje corporal que rodea al discurso (Lambert, 1981). Tanto en CorBPG como en el CorXIX vemos estos aspectos. En Galdós, por ejemplo, ya hemos mostrado casos relacionados con el modo de articulación, la actitud y el lenguaje gestual de los personajes en los ejemplos (2), (3) y (1), respectivamente. En relación con el lenguaje gestual vemos un uso notablemente mayor en el novelista canario que en el resto de autores de CorXIX. Para calibrarlo, hemos contado cuántas de las proposiciones proyectoras suspendidas a las que precede un vocativo tienen referencias al lenguaje gestual -es decir, casos como el ejemplo (1) mostrado en la introducción-. Como se advierte en la Tabla 3, Galdós recurre a este tipo de información de forma mucho más habitual (34,3\% de los ejemplos) que los autores que componen nuestro CorXIX (21,5\%).

Tabla 3

Distribución de proposiciones proyectoras con y sin referencias al lenguaje gestual de los personajes

\begin{tabular}{llllc}
\hline & CorBPG & $\%$ & CorXIX & $\%$ \\
\hline $\begin{array}{l}\text { Proposiciones proyectoras sin referencias } \\
\text { al lenguaje gestual de los personajes }\end{array}$ & 881 & 65,7 & 223 & 78,5 \\
\hline $\begin{array}{l}\text { Proposiciones proyectoras con } \\
\text { referencias al lenguaje gestual de los } \\
\text { personajes }\end{array}$ & 460 & 34,3 & 61 & 21,5 \\
\hline Total & 1.341 & 100 & 284 & 100 \\
\hline
\end{tabular}

Fuente: Elaboración propia. 
De estos resultados se puede extraer una serie de conclusiones que nos servirán de base para construir el análisis que se presenta a continuación. En primer lugar, hemos comprobado que a pesar de que Galdós recurre a la proyección del discurso de sus personajes utilizando estilo directo de forma mucho más habitual que el resto de autores con los que compartió plana, el uso de los vocativos en ambos corpus es similar (ligeramente superior en CorXIX, de hecho). A pesar de ello, sin embargo, observamos un patrón que descuella claramente en Galdós respecto de CorXIX: el uso de vocativo es mucho más frecuente cuando la proposición proyectora aparece suspendida. Además, en estos casos es también en CorBPG donde existe un mayor uso de referencias al lenguaje corporal de los personajes, uno de los recursos habituales a los que recurren los narradores en el empleo de suspensiones. Esta construcción -que se recoge en la Tabla 4 utilizando un ejemplo que analizamos en el apartado de objetos de la escena-, es en la que nos centramos en este artículo. En el análisis que sigue veremos que la relación entre los vocativos y las referencias al lenguaje gestual de los personajes no parece fruto de la casualidad, sino el resultado de patrones formales y funcionales estilísticamente significativos con los que Galdós crea distintos efectos, como la sensación de simultaneidad entre el lenguaje gestual del personaje y sus palabras y la caracterización o la construcción del universo ficticio que el autor nos plantea.

Tabla 4

Patrón estructural objeto de análisis

\begin{tabular}{lll}
\hline $\begin{array}{l}\text { Inicio proposición } \\
\text { proyectada (con } \\
\text { vocativo) }\end{array}$ & $\begin{array}{l}\text { Proposición proyectora } \\
\text { (con referencia a } \\
\text { lenguaje gestual) }\end{array}$ & $\begin{array}{l}\text { Final proposición } \\
\text { proyectada }\end{array}$ \\
\hline- Mujer. & $\begin{array}{ll}\text {-dijo Chinitas } \\
\text { cargando su escopeta- }\end{array}$ & quítate de en medio. \\
\hline
\end{tabular}

\section{Análisis: vocativos y lenguaje gestual}

La hipótesis sobre la que se construye nuestro análisis es que Galdós recurre al vocativo como recurso estructural antes de una proposición proyectora. Esto es, el vocativo actúa como un elemento que permite separar el parlamento del personaje en dos e insertar, entre ambas partes, la proposición proyectora, en la que encontramos información estilísticamente relevante. En el ejemplo (4), "Mi General” y "tengo una 
gallina asada, y me parece que después de lo que hemos hecho, bien podemos comérnosla tranquilamente" podrían haber claramente aparecido juntas, con la proposición proyectora al final, como se muestra en (4bis), en el que hemos reordenado el texto original. Naturalmente, el primer segmento no tiene por qué contener solo un vocativo, sino que bien puede contemplar también otros elementos (como en el ejemplo (3) mostrado más arriba). Sin embargo, el vocativo es el denominador común de este patrón sobre el que se construye la primera parte del parlamento del personaje y el que permite suspender la proposición proyectora que introduce el discurso.

(4) - Mi General - dijo D. Marcelino Oraa, entrando presuroso y risueño-, tengo una gallina asada, y me parece que después de lo que hemos hecho, bien podemos comérnosla tranquilamente. [ $\mathrm{Lu}$ chana, capítulo 40]

(4bis.) - Mi General, tengo una gallina asada, y me parece que después de lo que hemos hecho, bien podemos comérnosla tranquilamente- dijo D. Marcelino Oraa, entrando presuroso y risueño.

Mediante este esquema se pueden conseguir diversos efectos. El primero y más claro es, sin duda, el de la simultaneidad. Las suspensiones permiten lograr "una impresión de simultaneidad entre el discurso y la información contextual descrita por el narrador, que a su vez puede sugerir similitudes con la coexistencia simultánea del habla y el lenguaje corporal en la vida real" (Mahlberg et al., 2013, p. 40) ${ }^{9}$. Por eso es habitual encontrar el lenguaje corporal en el que aquí nos centramos. El deseo de Galdós de ofrecer realismo parece ser la razón. Sirvan como botón de muestra los ejemplos (5) y (6). En el primero, por un lado, Bragas interpela a Chaperón al tiempo que lo aparta del grupo para hablarle en secreto. En (6), por su parte, Polito se acerca al grupo donde se encuentra León para interpelarle y pedirle el coche. En ambos casos, el vocativo que precede a la proposición proyectora permite que la acción de los personajes (en un caso distanciarse de un grupo, en otro caso distanciarse de él) aparezca interrumpiendo el discurso del personaje, lo que crea

9 La cita original es la siguiente: "an impression of simultaneity between the speech and the contextual information described by the narrator, which in turn can suggest similarities to the simultaneous occurrence of speech and body language in real life". La traducción es nuestra. 
la impresión de que tanto las palabras de Bragas y de Polito como sus acciones tienen lugar a la vez. Naturalmente, la forma del gerundio también contribuye a ello, pero es la interrupción en la secuencia de eventos narrados la que, debido al carácter lineal del texto, acentúa el efecto de simultaneidad (Nieto Caballero, 2018).

(5) - Amigo Chaperón - dijo el astuto Bragas con afabilidad y desviando un poco del grupo al Comisario para hablarle en secreto-, cuando hablo de amigos me refiero a personas. [El terror de 1824, capítulo 4]

(6) —Allí está León. ¡Eh!, ¡León! ¿Nos cedes tu coche? — dijo Polito, acercándose a un grupo donde había diputados y algún ministro-. ¿Nos cedes tu coche? [La familia de León Roch, capítulo 1]

La frecuencia de estos ejemplos parece indicar que el uso del vocativo antepuesto a una proposición proyectora con lenguaje gestual no es fruto de la casualidad, sino un recurso al que Galdós recurre deliberadamente con frecuencia para dividir el parlamento del personaje en dos y ofrecer información en la proposición proyectora con la que lograr el mencionado efecto de sincronía. Este uso se refuerza si tenemos en cuenta que, sobre este patrón estructural tan marcado de "inicio proposición proyectada (con vocativo) + proposición proyectora (con referencia a lenguaje gestual) + final proposición proyectada" existen además otros procedimientos repetidos en la forma en la que Galdós emplea el lenguaje gestual. Tres de los más habituales son:

- las referencias explícitas a partes del cuerpo concretas,

- las referencias a objetos de la escena en la que se desarrolla la acción y

- las referencias espaciales que contribuyen a definir la propia escena.

Además de favorecer el efecto de sincronía que acabamos de explicar, estos elementos pueden contribuir a caracterizar a los personajes e incluso construir el universo ficticio que el autor plantea, como explicamos a continuación.

\section{Partes del cuerpo}

En las proposiciones proyectoras suspendidas y precedidas por vocativos hemos encontrado referencias de hasta dieciséis partes del cuerpo distintas: frente, manos, hombros, ojos, piernas, pies, cejas, ceño, 
pecho, codo, dedos, rodillas, cuello, boca, lengua, rostro. Algunas son muy recurrentes y en ellas se advierten usos repetidos que desempeñan funciones concretas. Un comportamiento habitual es que el personaje cuyo discurso se introduce ponga las manos en los hombros del interpelado, como se puede observar en (7). Como ya se ha señalado en otros estudios, esta es una práctica de la que Galdós se vale habitualmente para reforzar el afecto de un personaje hacia otro (Nieto Caballero, 2019b). El ejemplo (7) es un caso muy claro de esta función, donde Miquis (cuya intervención se introduce) utiliza el contacto corporal para consolar a su amigo Lope, interpelado en la primera parte del parlamento mediante un vocativo. Tal uso, además, es propio de Dickens (Mahlberg, 2013), y bien podría servir para reforzar su mencionada influencia en nuestro autor, pues el novelista victoriano del mismo modo incluye el lenguaje corporal en las proposiciones que introducen discurso (Nieto Caballero, 2019a).

(7) - Amigo D. Lope- dijo, poniendo sus dos manos sobre los hombros del caballero, que parecía más muerto que vivo-, hemos llegado a lo que yo me temía. [Tristana, capítulo 22]

Con otras partes del cuerpo encontramos usos igualmente significativos. En (8), por ejemplo, Torquemada se lleva el pañuelo a los ojos, lo cual ya ha sido identificado en Galdós desempeñando una función particular. El hecho de llevarse el pañuelo a los ojos es un bloque textual útil para expresar la tristeza de personajes masculinos (Nieto Caballero, 2019a). El ejemplo (8) es una muestra perfecta de esto, en el cual la tristeza de Torquemada se expresa en la proposición proyectora mediante la referencia al lenguaje gestual, a la que precede otro vocativo ("hija"). Ejemplos como este sirven para caracterizar a los personajes y transmitir actitud. Otro caso similar sería (9). No es una referencia explícita a los ojos, pero sí a la mirada. Las miradas son muy significativas en el género novelesco (Korte, 1997), pues pueden ayudar además a lograr efectos de autenticidad a través de la simultaneidad del habla de los personajes y la comunicación no verbal. El ejemplo (9) es una buena muestra. Como (8), sirve para proyectar una actitud — que en este caso se nos ofrece explícitamente mediante el adverbio "severísimamente" - y una vez más aparece introducida por un vocativo que parece ayudar a partir el parlamento en dos e insertar la proposición proyectora entre ambas mitades, lo 
que contribuye a crear la simultaneidad entre la comunicación verbal y no verbal del personaje.

(8) -Sí, hija, a la Virgen del Carmen - dijo Torquemada, llevándose el pañuelo a los ojos-. Me parece muy bien. Cada uno empuje por su lado, a ver si entre todos... [Torquemada en la hoguera]

(9) - Muy bien, Sr. D. Maximiliano, muy bien-dijo doña Lupe mirando severísimamente a su sobrino-. Siéntate que hay para rato. [Fortunata y Jacinta, capítulo 2]

Por último, las referencias a partes del cuerpo pueden servir para lograr una caracterización más directa. Tal sería el caso de (10), donde, aunque no se trata de una parte del cuerpo como tal, sí es un rasgo distintivo y único de la constitución de Eliseo Martínez en Misericordia, cuya memorabilidad estriba precisamente en que, además de manco, es cojo. La pata de palo dota al personaje de individualidad en la historia. El hecho de incluir esta información en una suspensión aísla el rasgo que lo individualiza y enfatiza (Mahlberg y Smith, 2012). Y la suspensión de nuevo es tal porque se ha fragmentado el parlamento del personaje en dos y en la primera parte se incluye un vocativo (señoras). Es decir, además de simultaneidad, hay caracterización de los personajes, no solo transmitiendo actitudes, como en los ejemplos (8) a (9), sino mediante la inserción de rasgos concretos.

(10) - Ea, que estamos en la casa de Dios, señoras - dijo Eliseo dando golpes en el suelo con su pata de palo-. Guarden respeto y decencia unas para otras, como manda la santísima dotrina. [Misericordia, capítulo 4]

\section{Objetos de la escena}

Otro elemento recurrente en las descripciones de lenguaje gestual son los objetos de la escena, con los que no solo se configura el universo ficticio que el autor nos plantea, sino que además pueden ayudar a caracterizar a los personajes por el significado que adquieren. De nuevo, estas referencias se encuentran con frecuencia indisolublemente unidas al uso de vocativos. Y, otra vez de nuevo, este parece ser el elemento al que Galdós recurre para poder dividir el parlamento del personaje en dos e introducir entre ambos segmentos la descripción del lenguaje gestual del personaje, que en esta posición parece enfatizarse. Las re- 
ferencias a objetos son muy dispares. A veces se relacionan con las partes del cuerpo que acabamos de comentar en el apartado anterior. Tal es el caso de (11). Como se puede observar, la referencia a la cabeza y la nariz del personaje se relaciona con el tapete de la mesa para indicar un movimiento. Además de crear la impresión de simultaneidad entre el discurso del personaje y sus movimientos, en este caso concreto el gesto ayuda a proyectar el rol de servidumbre de Elías, reforzando así el valor de sus palabras de sumisión. Sin duda, esto ayuda a proyectar su caracterización como un personaje manso y disciplinado.

(11) - Señor - dijo Elías humillando su cabeza hasta tocar con la nariz el tapete de la mesa-, yo no sé cómo V. M. no se cansa de protegerme. Yo, que jamás oculto la verdad a V. M., me atrevo a decirle respetuosamente que mi sobrinillo no merece semejante favor. Es un loco: tiene la cabeza llena de desatinos, y creo que jamás será un hombre formal. Si me atreví a pedir a V. M. ese favor, fue por los servicios que ha prestado el chico a nuestra santa causa, uniéndose a esos admirables, aunque indirectos, instrumentos de justicia que esta noche van a salvar a la patria. [La Fontana de oro, capítulo 41]

En ocasiones, el valor de los objetos a la hora de transmitir una idea es más claro, pues los propios tienen un valor simbólico en sí mismo sin necesidad de asociarse con acciones concretas. Tal es el caso de las armas, cuya mención en una proposición proyectora sirve normalmente para reforzar el carácter violento del personaje que hace uso del vocativo. Sirva como apoyo de lo que decimos el ejemplo (12). Como se puede advertir, la sincronía entre el discurso del personaje y sus movimientos se construye sobre el eje de las armas, que refuerzan el discurso violento del personaje, introducido siempre por un vocativo que también ayuda a definir esta violencia mediante la interpelación directa a otro personaje.

(12) - Mujer - dijo Chinitas cargando su escopeta- quítate de en medio. [El 19 de marzo y el 2 de mayo, capítulo 26]

Aunque no se trate de un arma, el cuchillo del ejemplo (13) desempeña una función similar. Se trata, además, del mismo patrón estructural, pues la primera parte del parlamento del personaje contiene únicamente un vocativo que indica vehemencia. La llamada de atención 
a Domiciana se combina con la referencia al cuchillo, lo que sirve para reforzar la amenaza de Lucila y su carácter violento. Podría decirse que las referencias a objetos actúan como bloques textuales con los que crear significados específicos —en el caso concreto de este ejemplo el de la proyección del carácter violento del personaje con el que se asocian-. Este valor se acentúa mediante el modelo estructural en el que se encuentran, pues aparecen indisolublemente unidos al vocativo del discurso del personaje. La llamada de atención de este, en combinación con el objeto (y el significado que adquiere) sirve para amplificar el efecto de violencia que tanto el vocativo como el objeto ya tienen, aunque en menor grado, por separado.

(13) —Domiciana - dijo apretando el mango del cuchillo-, si crees que ahora jugarás también conmigo, te equivocas... No vengo por dedadas de miel, sino por verdades. [Los duendes de la camarilla, capítulo 21]

\section{Referencias espaciales}

Por último, en las proposiciones proyectoras precedidas por vocativos también es frecuente encontrar referencias espaciales. En la novela realista española, el espacio se erige como un elemento de importancia cardinal en la ilusión de transparencia y el efecto de verosimilitud que domina este movimiento (Martínez Carazo, 2006). En el caso de Galdós, además, el espacio adquiere un valor simbólico en relación con determinadas funciones que envuelven y llegan a definir la actuación de los personajes y también actúa como uno de los ejes vertebradores en la creación de los universos ficticios (Nieto Caballero, 2019b). Este último uso es el que observamos precisamente en las referencias espaciales de las proposiciones proyectoras. Otra vez el vocativo parecer ser el recurso al que Galdós acude para dividir el parlamento del personaje en dos con el objeto de insertar la referencia espacial en la proposición proyectora y enfatizar el espacio en el que se desarrolla la acción verbal. Un ejemplo habitual son las calles, tal como advertimos en (14), donde la proposición proyectada precedida por vocativo, además de lograr un efecto de simultaneidad entre el discurso del personaje y su movimiento, la referencia a la calle de San Gil sirve para posicionar lugares en los que se desarrolla la acción mediante su enclave en una vía determinada. Este empleo encaja con el apuntado en otros 
estudios sobre el hábito galdosiano de recurrir a tales lugares para situar la acción presentada en un espacio real, de manera que con ello logra un alto grado de verosimilitud en el marco del paisaje urbano en el que transcurre la mayoría de sus historias (Nieto Caballero, 2019b).

(14) - iEh, padre Luengo! - dijo Montoria llamando al fraile de este nombre, que entraba apresuradamente en la calle de San Gil-. ¿Qué hay? ¿Dónde está el Capitán general? ¿Ha perecido entre las ruinas? [Zaragoza, capítulo 28]

Además de las calles, las puertas también son habituales. En general, en Galdós se trata ante todo de ejemplos de punto de emplazamiento, pues suelen actuar como la referencia en la que se sitúan los personajes y en torno a la que se construye la escena que se nos presenta (Nieto Caballero, 2019b). En el caso de los ejemplos localizados en proposiciones proyectoras antepuestas a vocativos, además, las referencias sirven no solo para definir el universo ficticio, sino para lograr un efecto de simultaneidad, pues se asocian habitualmente a verbos que indican movimiento. Sirva como ejemplo (15), en donde la puerta se erige como referencia sobre la que se construye el movimiento de Fortunata.

(15) -Dispénseme usted, amigo D. Evaristo- dijo Fortunata apareciendo en la puerta del gabinete, con bata de diario, un delantal muy grande y pañuelo liado a la cabeza-. Estoy de limpia. [Fortunata y Jacinta, parte 3, capítulo 4]

La combinación del efecto de simultaneidad con la construcción del universo ficticio mediante el uso de referencias espaciales refuerza el dominio del espacio visual y el movimiento de Galdós, que suele ser considerado por la crítica como el recurso con el que "se prefigura el ritmo cinematográfico" (Arroyo Díez, 2011, p. 366) que caracteriza su estilo. Los ejemplos localizados en este sentido sin duda refuerzan esta hipótesis.

Por otra parte, las muestras de referencias espaciales, unidas a las partes del cuerpo y a los objetos que ya hemos analizado en los apartados anteriores demuestran, en definitiva, que existen manejos repetidos y bien definidos dentro del patrón objeto de análisis de este artículo - las proposiciones proyectoras con referencias a lenguaje gestual precedidas por un vocativo-. A la luz del valor de los ejemplos 
que hemos ido desgranando a lo largo del artículo, podemos concluir que el vocativo contiene un claro valor estructural en la producción narrativa de Galdós. Más allá de ser un recurso empleado por personajes para llamar la atención de otros, desde un punto de vista narrativo el novelista recurre a este elemento con maestría para configurar un esquema estructural con el que crear distintos efectos, tales como recurso fragmentador del discurso del personaje, lo que permite colocar la proposición que proyecta el discurso entre ambos segmentos resultantes y lograr efectos como la simultaneidad del discurso y el lenguaje corporal de los personajes, enfatizar aspectos relacionados con la caracterización o construir el universo ficticio mediante referencias espaciales que ayudan a definir la escena.

\section{Conclusiones}

El vocativo es un elemento mediante el cual se logran distintos efectos relevantes desde un punto de vista literario. En este artículo hemos analizado la presencia de este recurso en la producción novelística de Galdós. Para ello, hemos recurrido a un corpus constituido por su producción narrativa (CorBPG), y hemos cotejado la recurrencia de ese patrón con la frecuencia con la que se emplea en un corpus de referencia (CorXIX) formado por la producción narrativa de ocho de los autores más representativos del realismo decimonónico español. Como se ha podido comprobar, el empleo de vocativos en CorBPG y CorXIX es similar. Sin embargo, en la producción de Galdós su presencia es sensiblemente mayor cuando la proposición proyectora que lo introduce se encuentra suspendida. Además, en este patrón se advierte también una mayor recurrencia a referencias al lenguaje corporal de los personajes en CorBPG que en CorXIX, que se materializan dentro de un hábito estilísticamente significativo en su producción narrativa, pues parece existir una relación directa entre el uso del vocativo y las referencias al lenguaje gestual del personaje en la proposición proyectora que lo introduce. En concreto, el vocativo actúa como un elemento que permite separar el parlamento del personaje en dos e insertar, entre ambas partes, la proposición proyectora con información estilísticamente relevante que permite crear distintos efectos. El más inmediato es, sin duda, el de la impresión de simultaneidad entre las palabras del propio personaje y su lenguaje corporal. Sin embargo, las referencias al lenguaje gestual también pueden desempeñar funciones más específi- 
cas relacionadas con la caracterización o la construcción de universos ficticios que el autor nos plantea, como hemos visto mediante el empleo de referencias explícitas a partes del cuerpo, a objetos concretos o a referencias espaciales que definen la escena.

En el artículo hemos seleccionado ejemplos de distintas novelas con el fin de demostrar que no se trata de un fenómeno aislado, sino de un patrón estable a lo largo de la producción del autor. Estos han podido ser localizados gracias al enfoque de estilística de corpus que hemos adoptado para llevar a cabo el análisis. En este sentido, podría decirse que, además de analizar el uso del vocativo en la producción narrativa de Galdós, también hemos pretendido ilustrar el valor de los enfoques de corpus en el análisis de textos en lengua española en general, un área todavía escasa de estudios que adopten este tipo de metodologías.

\section{Referencias bibliográficas}

Alonso-Cortés, Á. (1999). Las expresiones exclamativas. La interjección y las expresiones vocativas. En I. Bosque y V. Demonte (Eds.), Nueva gramática descriptiva de la lengua española, vol. 3 (pp. 3993-4213). Espasa-Calpe.

Arroyo Díez, M. C. (2011). Aspectos espaciales y visuales en las primeras novelas contemporáneas Benito Pérez Galdós y su repercusión en la novela española actual [Tesis de doctorado no publicada]. Universidad de Valladolid.

Balbín de, R. (1968). Sobre la oración vocativa en las 'Rimas' de G.A. Bécquer. En A. Quilis, R. B. Carril, y M. Cantarero (Eds.), XI Congreso Internacional de Lingüistica y Filología Románicas: Actas, vol. 4 (pp. 1761-1768). Revista de Filología Española.

Bañón Hernández, A. M. (1993). El vocativo en español. Propuestas para su análisis lingüístico. Octaedro.

Bravo, D. y Briz, A. (Eds.). (2004). Pragmática sociocultural: estudios sobre el discurso de cortesía en español. Ariel.

Brioso Sánchez, M. (1971). El vocativo y la interjección. Habis, (2), 35-48.

Busse, B. (2006). Vocative constructions in the language of Shakespeare. John Benjamins.

Cuenca, M. J. (2013). Uso del vocativo en la entrevista política: género discursivo y (des)cortesía. Discurso y Sociedad, 7(3), 
522-552. http://www.dissoc.org/ediciones/v07n03/DS7(3) Cuenca.pdf

Edeso Natalías, V. (2005). Usos discursivos del vocativo en español. Español actual: Revista de español vivo, (84), 123-142.

Halliday, M. A. K. (2004). An introduction to functional grammar. Arnold.

Korte, B. (1997). Body language in literature. University of Toronto Press.

Lambert, M. (1981). Dickens and the suspended quotation. Yale University Press.

Leech, G. N. (1983). Pragmatics. Longman.

Levy, M. (1937). La influencia de Dickens en Galdós. Columbia University.

Mahlberg, M., Smith, C., y Preston, S. (2013). Phrases in literary contexts: Patterns and distributions of suspensions in Dickens's novels. International Journal of Corpus Linguistics, 18(1), 3556. https://doi.org/10.1075/ijcl.18.1.05mah

Mahlberg, M. y Smith, S. (2012). Dickens, the suspended quotation and the corpus. Language and Literature, 21(1), 51-65. https:// doi.org/10.1177/0963947011432058

Mahlberg, M. (2016). Corpus stylistics. En V. Sotirova (Ed.), The Bloomsbury Companion to Stylistics (pp. 139-156). Bloomsbury.

Mahlberg, M. (2014). Corpus stylistics. En M. Burke (Ed.), The Routledge Handbook of Stylistics (pp. 387-392). Routledge.

Mahlberg, M. (2013). Corpus stylistics and Dickens's Fiction. Routledge.

Martínez Carazo, C. (2006). De la visualidad literaria a la visualidad filmica. "La Regenta" de Leopoldo Alas "Clarín". Llibros del Pexe.

McGovern, T. (2000). Dickens in Galdós. Peter Lang.

McIntyre, D. y Walker, B. (2019). Corpus stylistics: Theory and practice. Edinburgh University Press.

Monserrat Roig, C. (2009). Los vocativos metafóricos en las comedias de Plauto. Cuadernos de filología clásica: estudios latinos, 30(1), 7-26.

Newsom, R. (2000). Style of Dickens. En P. Schlicke (Ed.), The Oxford Reader's Companion to Charles Dickens (pp. 553-557). Oxford University Press. 
Nieto Caballero, G. (2019a). Análisis de la influencia de Charles Dickens en el estilo de Benito Pérez Galdós a través del lenguaje gestual de sus personajes: un estudio de corpus. Dicenda. Estudios de Lengua y Literatura Españolas, (37), 321-341. https://doi.org/10.5209/dice.65006

Nieto Caballero, G. (2019b). El espacio como eje vertebrador en la creación del universo ficticio galdosiano: un estudio de corpus. Signa, (28), 1203-1238. https://doi.org/10.5944/signa. vol28.2019.25116

Nieto Caballero, G. (2018). Metodologías de corpus en el análisis de textos literarios en lengua española: el ejemplo de Pérez Galdós. Estudios Humanísticos. Filología, (40), 373-391. https://doi.org/10.18002/ehf.v0i40.5158

Pattison, W. T. (1954). Benito Pérez Galdós and the creative process. University of Minnesota Press.

Scott, M. (2013). WordSmith Tools Manual. Version 6.0. Lexical Analysis Software.

Tambling, J. (2013). Dickens and Galdós. M. Hollington (Ed.), The Reception of Charles Dickens in Europe (pp. 191-196). Bloomsbury.

Universidad de Alicante. (15 de septiembre de 2019). Repositorio digital de la Biblioteca Virtual Miguel de Cervantes. http://www. cervantesvirtual.com/ 